Exploring biorthonormal transformations of pair-correlation functions in atomic structure variational calculations

This article has been downloaded from IOPscience. Please scroll down to see the full text article.

2010 J. Phys. B: At. Mol. Opt. Phys. 43074017

(http://iopscience.iop.org/0953-4075/43/7/074017)

View the table of contents for this issue, or go to the journal homepage for more

Download details:

IP Address: 130.235.64.24

The article was downloaded on 17/07/2010 at 12:24

Please note that terms and conditions apply. 


\title{
Exploring biorthonormal transformations of pair-correlation functions in atomic structure variational calculations
}

\author{
S Verdebout ${ }^{1}$, P Jönsson ${ }^{2}$, G Gaigalas $^{3}$, M Godefroid ${ }^{1}$ and \\ C Froese Fischer ${ }^{4}$ \\ ${ }^{1}$ Chimie Quantique et Photophysique, CP160/09, Université Libre de Bruxelles, Av. F.D. Roosevelt 50, \\ B-1050 Brussels, Belgium \\ ${ }^{2}$ Nature, Environment, Society, Malmö University, 205-06 Malmö, Sweden \\ ${ }^{3}$ Vilnius University Research Institute of Theoretical Physics and Astronomy, A. Goštauto 12, \\ LT-01108 Vilnius, Lithuania \\ ${ }^{4}$ National Institute of Standards and Technology, Gaithersburg, MD 20899-8420, USA \\ E-mail: mrgodef@ulb.ac.be
}

Received 15 September 2009, in final form 6 November 2009

Published 19 March 2010

Online at stacks.iop.org/JPhysB/43/074017

\begin{abstract}
Multiconfiguration expansions frequently target valence correlation and correlation between valence electrons and the outermost core electrons. Correlation within the core is often neglected. A large orbital basis is needed to saturate both the valence and core-valence correlation effects. This in turn leads to huge numbers of configuration state functions (CSFs), many of which are unimportant. To avoid the problems inherent to the use of a single common orthonormal orbital basis for all correlation effects in the multiconfiguration Hartree-Fock (MCHF) method, we propose to optimize independent MCHF pair-correlation functions (PCFs), bringing their own orthonormal one-electron basis. Each PCF is generated by allowing single- and double-excitations from a multireference (MR) function. This computational scheme has the advantage of using targeted and optimally localized orbital sets for each PCF. These pair-correlation functions are coupled together and with each component of the MR space through a low dimension generalized eigenvalue problem. Nonorthogonal orbital sets being involved, the interaction and overlap matrices are built using biorthonormal transformation of the coupled basis sets followed by a counter-transformation of the PCF expansions. Applied to the ground state of beryllium, the new method gives total energies that are lower than the ones from traditional complete active space (CAS)-MCHF calculations using large orbital active sets. It is fair to say that we now have the possibility to account for, in a balanced way, correlation deep down in the atomic core in variational calculations.
\end{abstract}

\section{Introduction}

There are several widely used methods for calculating manyelectron atoms, but many-body effects still represent a real challenge. The many-body perturbation theory (MBPT) appears to be the most effective for atoms with one valence electron but its accuracy is not satisfactory for the atoms with more than one valence electron, mainly because of the poor convergence of the MBPT for the valence-valence correlations [1]. However, the core-valence correlations still can be effectively treated with MBPT. For this reason it was suggested to combine many-body perturbation theory for the core-valence correlations with the configuration interaction (CI) for valence-valence correlations within the (CI+MBPT) method [2], but this extension to atoms with more than three electrons in open shells met some difficulties. A method based on the so-called $V^{N-M}$ approximation has been proposed by Dzuba and Flambaum [3] including core-valence correlations by means of MBPT. The most recent extension of MBPT is the development of a configuration-interaction plus all-order method for atomic calculations [4]. This is a theoretical method combining the all-order approach currently used in 
precision calculations of the properties of monovalent atoms with the configuration interaction approach that is applicable for many-electron systems.

The coupled-cluster (CC) approach [5] is an interesting alternative, summing up all orders and taking into account pair correlations. Interesting computational developments have been proposed [6, 7], not only in physics but also in quantum chemistry (for a complete review, see [8]). Successful applications do exist [9-11], with some limitations for atoms with more complicated electron structures than alkali atoms. According to [2], the most obvious shortcoming of the $\mathrm{CC}$ method is the neglect of three-particle correlations. Moreover, it treats the valence-valence and core-valence correlations at the same level of approximation, while the former is much stronger than the latter.

In quantum chemistry, variational complete active space self-consistent field (CASSCF) methods are quite successful for describing small and medium-size molecules but are not sufficient when external (dynamic) correlation must be included [12]. The latter are treated through secondorder perturbation theory using a single or multireference state [13, 14]. Nonperturbative variational methods treat many-body effects in an accurate way for valence electrons, in both nonrelativistic and relativistic schemes. In this line, multiconfigurations Hartree-Fock (MCHF) and DiracHartree-Fock (MCDHF), often combined with CI methods $[15,16]$, have been widely used for accurate calculations of many-electron atomic properties, focusing on the valence and core-valence correlation of large atomic systems. The accuracy of CI is limited by the incompleteness of the set of configurations used if the one-electron orbital basis is complete. A practical limitation is the number of possible configuration state functions (CSFs) that becomes so large for a many-electron atom that one has to select only a small fraction of them [17]. This is often done by neglecting core excitations or only including a limited number of them $[18,19]$. The CI method is by definition (orbital) basis dependent, while the multiconfiguration methods are not; the CI problem being iteratively coupled to the orbital optimization [20, 21]. As discussed in section 4, the shape of the resulting orbitals strongly depends on the type of correlation introduced through the multiconfiguration expansion [22]. This is due to the properties of the variational principle applied for deriving the MCHF/MCDHF equations to be solved, with the consequence that a set of radial distributions resulting from a given correlation model/expansion could become inadequate, or at least incomplete, for another model or for an extension of the original one. Other problems encountered in variational multiconfiguration calculations are discussed in the present work, illustrating the difficulty of optimizing a single orthonormal orbital basis set from which CSF expansions would describe efficiently all correlation effects for a given physical state, and would produce reliable expectation values for any operator other than the Hamiltonian.

To avoid these problems inherent to the use of a single common orthonormal orbital basis for all correlation effects in the MCHF method, we propose to first optimize independent
MCHF pair correlation functions (PCFs), bringing their own orthonormal one-electron basis. These PCFs are then coupled to each other through a low-dimension generalized eigenvalue problem. A pioneer and inspiring work was done by Froese Fischer and Saxena [23, 24] who introduced the separatedpair MCHF approach. The originality of the present study lies in the way the one-electron nonorthogonalities are treated for setting up the interaction matrix between the reference and the PCF spaces. For each coupling matrix element, we adopt the biorthogonal orbital transformations and CI eigenvectors counter-transformations, as originally proposed by Malmqvist [25] and later extended to the spherical atomic symmetry by Olsen et al [26].

The PCF interaction approach is tested, in the nonrelativistic approximation, on the ground state of beryllium, using a multireference description for the valence electrons. The results are compared with the complete active space (CAS)-MCHF method.

\section{The variational method}

The MCHF method, that is extended and explored with respect to nonorthogonalites in the current work, can be derived from the variational principle. A state $\Psi$ is an eigenstate of the Hamiltonian $H$ if and only if the energy functional

$$
E[\Psi] \equiv \frac{\langle\Psi|H| \Psi\rangle}{\langle\Psi \mid \Psi\rangle}
$$

is left unchanged for any infinitesimal variation in the state at the point $\Psi$. For the ground state or states that are the lowest of their symmetry, the variational method gives a minimum principle:

$$
E_{0} \leqslant \frac{\langle\Psi|H| \Psi\rangle}{\langle\Psi \mid \Psi\rangle},
$$

$E[\Psi]$ being the upper bound to the exact ground state energy $E_{0}$. Using a superposition ansatz

$$
|\Psi\rangle=\sum_{i=1}^{M} c_{i}\left|\Phi_{i}\right\rangle
$$

for describing the model state in the subspace of the $M$ basis states $\Phi_{i}$, the variational parameters $\left\{c_{i}\right\}$ can be determined by solving the generalized eigenvalue problem

$$
\mathbf{H c}=E \mathbf{S c},
$$

with $H_{i, j} \equiv\left\langle\Phi_{i}|H| \Phi_{j}\right\rangle$ and $S_{i, j} \equiv\left\langle\Phi_{i} \mid \Phi_{j}\right\rangle$ being, respectively, the Hamiltonian and overlap matrices. The total energies are found as roots of the secular equation

$$
\operatorname{det}(\mathbf{H}-E \mathbf{S})=0 .
$$

The eigenfunctions $\left\{\Psi^{(k)}\right\}$ as a model subspace satisfying

$$
\left\langle\Psi^{(k)} \mid \Psi^{(l)}\right\rangle=\delta_{k, l}, \quad\left\langle\Psi^{(k)}|H| \Psi^{(l)}\right\rangle=\epsilon_{k} \delta_{k l}
$$

are useful for the description of excited states since they according to the Hylleraas-Undheim theorem [27, 28] satisfy the conditions

$$
E_{k} \leqslant \epsilon_{k}, \quad \forall k=1, \ldots, M .
$$

That is, approximate eigenvalues obtained by diagonalizing the Hamiltonian in a subspace can only be stabilized when the latter is enlarged. 


\section{The nonrelativistic multiconfiguration Hartree-Fock method}

Starting from the nonrelativistic Hamiltonian for an $\mathrm{N}$-electron system

$$
H=\sum_{i=1}^{N}\left[-\frac{1}{2} \nabla_{i}^{2}-\frac{Z}{r_{i}}\right]+\sum_{i<j}^{N} \frac{1}{r_{i j}},
$$

the MCHF approach determines an approximate wavefunction $\Psi$ for the state labelled $\gamma L S$ of the form

$$
|\Psi(\gamma L S)\rangle=\sum_{i=1}^{M} c_{i}\left|\Phi\left(\gamma_{i} L S\right)\right\rangle,
$$

where $\gamma$ represents the dominant configuration and any additional quantum numbers required for uniquely specifying the state being considered. The CSFs $\left\{\Phi\left(\gamma_{j} L S\right)\right\}$ are built from a basis of one-electron spin orbitals

$$
\phi\left(n l m_{l} m_{s}\right)=\frac{1}{r} P(n l ; r) Y_{l m_{l}}(\theta, \varphi) \chi_{m_{s}},
$$

where the radial distributions $\{P(n l ; r)\}$ are to be determined. By applying the variational principle one obtains a set of integro-differential MCHF equations:

$$
\begin{aligned}
& \left\{\frac{\mathrm{d}^{2}}{\mathrm{~d} r^{2}}+\frac{2}{r}[Z-Y(n l ; r)]-\frac{l(l+1)}{r^{2}}-\epsilon_{n l, n l}\right\} P(n l ; r) \\
& =\frac{2}{r} X(n l ; r)+\sum_{n^{\prime} \neq n} \epsilon_{n l, n^{\prime} l} P\left(n^{\prime} l ; r\right)
\end{aligned}
$$

for the unknown radial distributions [15]. The equations are coupled to each other through the direct $Y$ and exchange $X$ potentials and the Lagrange multipliers $\epsilon_{n l, n^{\prime} l}$. The Lagrange multipliers force the radial orbitals to be orthonormal within the same $l$ subspace. Under these conditions the configuration state functions are orthonormal:

$$
\left\langle\Phi\left(\gamma_{i} L S\right) \mid \Phi\left(\gamma_{j} L S\right)\right\rangle=\delta_{i, j} .
$$

The mixing coefficients $\left\{c_{i}\right\}$ appearing in the expansion over CSFs also enter in the form of the potentials and can be determined by solving the CI problem

$$
\mathbf{H c}=E \mathbf{c}
$$

for the current set of radial distributions. The MCHF and CI problems are solved iteratively until self-consistency is reached for the radial distributions and for the selected CIeigenvector.

The multiconfiguration method incorporates an extension allowing radial nonorthonormality, which sometimes can be used to advantage. Evaluation of matrix elements when orbitals are nonorthogonal is complex, in general. In order to keep the energy expressions manageable a number of restrictions are imposed: the orbitals within each CSF are mutually orthogonal, there are at most two subshells in $\Phi\left(\gamma_{i} L S\right)$ containing spectator electrons whose orbitals are nonorthogonal to orbitals in the interacting function $\Phi\left(\gamma_{j} L S\right)$, if all the spectator electrons with nonorthogonal orbitals have the same $l$ value, then there are at most two such electrons in each of $\Phi\left(\gamma_{i} L S\right)$ and $\Phi\left(\gamma_{j} L S\right)$, the use of nonorthogonal orbitals is such that $\left\langle\Phi\left(\gamma_{i} L S\right) \mid \Phi\left(\gamma_{j} L S\right)\right\rangle=\delta_{i, j}$. Here the term spectator refers to those electrons not directly involved in the interaction [15]. Despite these restrictions, nonorthogonal orbitals have successfully been applied in a number of cases, describing major correlation effects in a very compact way $[22,29]$.

\section{Correlation and spatial location of orbitals}

The MCHF method results from the application of the variational principle, and the solution depends strongly on the energy functional or CSF expansion used to derive the MCHF equations. The direct influence of the CSF expansion on the shape of the resulting radial orbital basis can be exploited to target specific effects, but could also bring undesirable distortions of the wavefunction for the property of interest. To illustrate these effects let us look at the beryllium $1 \mathrm{~s}^{2} 2 \mathrm{~s}^{2}{ }^{1} \mathrm{~S}$ ground state. The valence pair-correlation function (PCF) is described by considering all possible excitations of the valence electron pair:

$$
\begin{aligned}
& \left|\Lambda_{\mathrm{VV}}\right\rangle=\alpha_{1}\left|1 \mathrm{~s}^{2} 2 \mathrm{~s}^{2}{ }^{1} \mathrm{~S}\right\rangle \\
& \quad+\sum_{n} \alpha_{n}\left|1 \mathrm{~s}^{2} 2 \mathrm{~s} n \mathrm{~s}{ }^{1} \mathrm{~S}\right\rangle+\sum_{n l, n^{\prime} l^{\prime}} \alpha_{n l, n^{\prime} l^{\prime}}\left|1 \mathrm{~s}^{2} n l n^{\prime} l^{\prime}{ }^{1} \mathrm{~S}\right\rangle .
\end{aligned}
$$

The core-valence PCF is obtained by promoting one core electron together with one valence electron:

$\left|\Lambda_{\mathrm{CV}}\right\rangle=\beta_{1}\left|1 \mathrm{~s}^{2} 2 \mathrm{~s}^{2}{ }^{1} \mathrm{~S}\right\rangle+\sum_{n l, n^{\prime} l^{\prime}} \beta_{n l, n^{\prime} l^{\prime}}\left|1 \mathrm{~s} 2 \mathrm{~s} n l n^{\prime} l^{\prime 1} \mathrm{~S}\right\rangle$.

Finally, the core-core PCF takes into account the correlation within the core:

$$
\begin{aligned}
& \left|\Lambda_{\mathrm{CC}}\right\rangle=\gamma_{1}\left|1 \mathrm{~s}^{2} 2 \mathrm{~s}^{2}{ }^{1} \mathrm{~S}\right\rangle \\
& \quad+\sum_{n} \gamma_{n}\left|1 \mathrm{~s} 2 \mathrm{~s}^{2} n \mathrm{~s}{ }^{1} \mathrm{~S}\right\rangle+\sum_{n l, n^{\prime} l^{\prime}} \gamma_{n l, n^{\prime} l^{\prime}}\left|2 \mathrm{~s}^{2} n l n^{\prime} l^{\prime}{ }^{1} \mathrm{~S}\right\rangle
\end{aligned}
$$

Each of the above sums represent correlation between a particular pair of electrons, namely $(2 s, 2 s)$ for valence correlation, $(1 \mathrm{~s}, 2 \mathrm{~s})$ for core-valence and $(1 \mathrm{~s}, 1 \mathrm{~s})$ for corecore correlation. For this reason they are called paircorrelation functions. As far as terminology is concerned, pair-correlation should be strictly used for double-excitations [30]. However, single-excitations are also included in our PCFs. Somewhat arbitrary we choose to include $\left|1 \mathrm{~s}^{2} 2 \mathrm{~s} n \mathrm{~s}^{1} \mathrm{~s}\right\rangle$ in the valence function and $\left|1 \mathrm{~s} 2 \mathrm{~s}^{2} n \mathrm{~s}^{1} \mathrm{~S}\right\rangle$ in the core-core function. Other choices are possible for the single-excitations. In the above case there is only one reference configuration, but applied to a multireference the PCFs capture major correlation effects in a very effective way $[15,31]$.

The dependence of the orbitals on the type of correlation is illustrated in figure 1. Here one clearly sees the contraction of the correlation orbitals when going from a valence to a corecore correlation calculation. Unfortunately, correlation effects are not additive, and interference occurs through multiple excitations. As we have described above, one can easily target specific correlation effects through the CSF expansion. However, the resulting set of radial distributions is localized in such a way that it becomes inadequate for representing other types of correlation. In our four-electron system it is for example not meaningful to use core-core correlation 

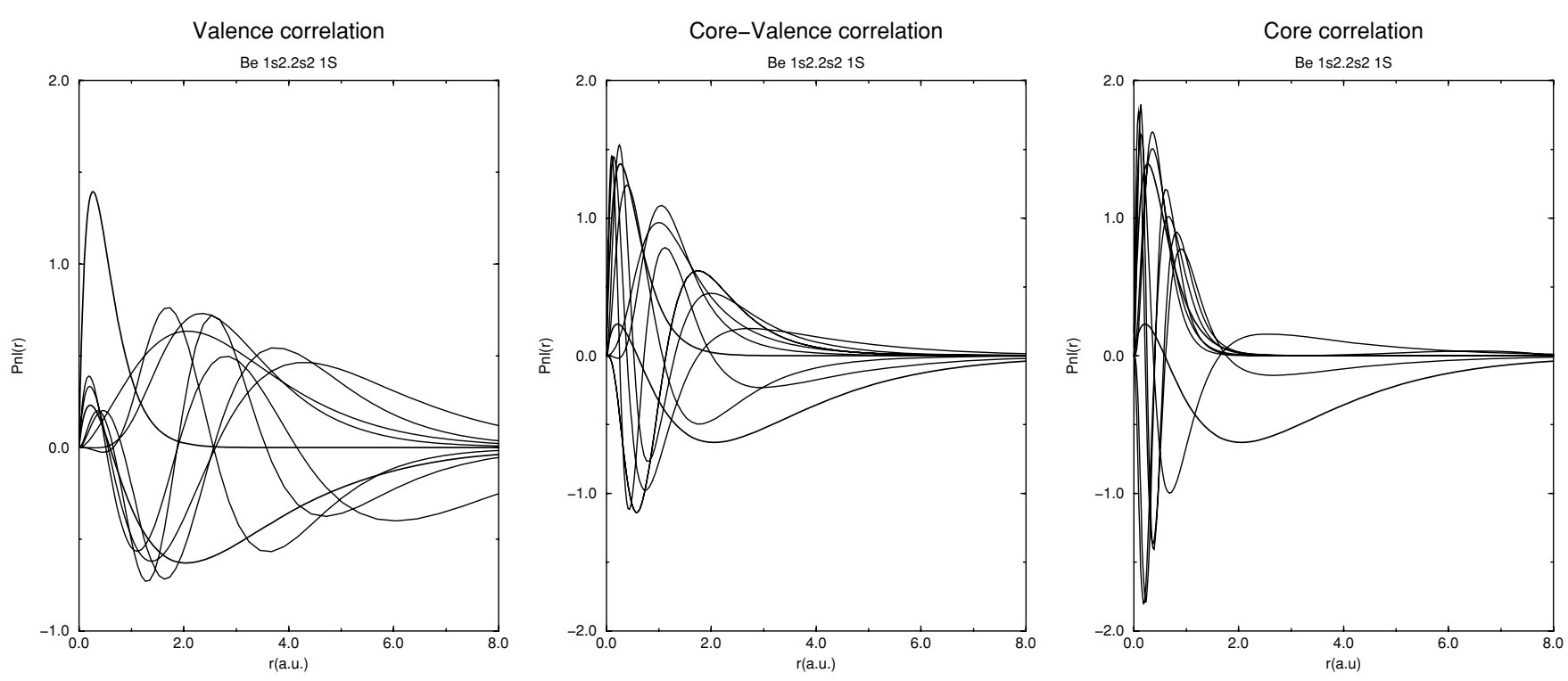

Figure 1. Contraction of the correlation orbitals when going from valence to core-valence and core-core correlation MCHF calculations of $\mathrm{Be} 1 \mathrm{~s}^{2} 2 \mathrm{~s}^{2} \mathrm{~S}$. The two thick lines correspond to the spectroscopic 1s (no node) and $2 \mathrm{~s}$ (one node) orbitals. Other lines represent the radial distributions of the correlation orbitals of the $n=4$ active set.

orbitals for describing valence correlation and vice versa. The problem with the space localization of the correlation orbitals becomes more pronounced for larger systems with many electron subshells.

Multiconfiguration expansions frequently target valence correlation and correlation between valence electrons and the outermost core electrons. Correlation within the core is neglected. Based on the combined valence and core-valence expansion, a single orbital basis is determined. While this approach gives orthonormal configuration state functions, and allows standard methods to be used for the construction of radial matrix elements, it has some drawbacks. A large orbital basis is needed to saturate both the valence and core-valence correlation effects. This in turn leads to huge numbers of CSFs, many of which are unimportant. Another problem is uneven convergence patterns as new layers of orbitals, dependent on the gain in variational energy, may be more or less contracted, affecting computed properties differently. Finally, there are difficulties to effectively incorporate correlation within the core. A solution to these problems, taking the nonorthogonal extension of the MCHF package all the way, would be to represent the wavefunction as an expansion over CSFs,

$$
|\Psi\rangle=\sum_{i=1}^{M} c_{i}\left|\Phi_{i}^{o p t}\right\rangle,
$$

where each CSF, depending on the correlation effect it describes, is built on an orbital set optimized for this effect. Since different orbital sets are involved, the CSFs are no longer orthonormal, and the expansion coefficients are obtained by solving the generalized eigenvalue problem. The Hamiltonian matrix elements coupling CSFs built on different and nonorthogonal orbital sets could be evaluated using their Slater determinant expansions and the Löwdin's cofactor method [32, 33], as adopted in many atomic structure packages [34-37]. For CSF expansions, a nonorthogonal extension of the underlying Racah algebra [38] is available [39, 40] but is not general enough. Moreover, both approaches quickly become prohibitive from a computational point of view.

\section{Pair-correlation function interaction calculations}

A different way of handling nonorthogonalities is offered by the biorthonormal transformation technique originally introduced by Malmqvist [25]. The transformation allows general matrix elements between PCFs to be computed fast and efficiently. To exploit this we represent the wavefunction not as an expansion over CSFs, but as an expansion over one or more reference CSFs $\left|\Phi_{i}^{r}\right\rangle$ and a number of known pair-correlation functions $\left|\tilde{\Lambda}_{j}\right\rangle$ built on separate and optimally localized orbital sets,

$$
|\Psi\rangle=\sum_{i=1}^{g} c_{i}\left|\Phi_{i}^{r}\right\rangle+\sum_{j=1}^{p} \tilde{c}_{j}\left|\tilde{\Lambda}_{j}\right\rangle .
$$

To avoid redundancies in the representation, reference CSFs are discarded from the PCFs. In the remaining sections we will use the notation $\left|\Lambda_{j}\right\rangle$ for PCFs including the reference CSFs and $\left|\tilde{\Lambda}_{j}\right\rangle$ for PCFs where the reference CSFs have been discarded (weights of reference CSFs have been set to zero). The Hamiltonian and overlap matrix elements between reference CSFs and PCFs and between different PCFs are computed using the biorthonormal transformation. The expansion coefficients $\left\{c_{i}, \tilde{c}_{j}\right\}$ are obtained by solving the corresponding generalized eigenvalue problem. The problem of computing a total wavefunction including valence, core-valence and core-core correlation thus reduces to a series of separate pair-correlation problems together with a relatively small generalized eigenvalue problem. Practically, the calculations proceed in the following steps.

(i) Perform an MCHF calculation for the reference expansion. 
(ii) Keep the orbitals from the first step fixed and perform separate MCHF calculations for the different PCFs including the reference.

(iii) Remove the reference CSFs from the PCFs by setting the weights to zero.

(iv) Loop over CSFs and PCFs perform biorthogonal transformations and evaluate the Hamiltonian and overlap matrix elements.

(v) Solve the generalized eigenvalue problem.

This computational scheme has the advantage of using targeted and optimally localized orbital sets for each of the PCFs. It is possible to include core correlation in a tractable way. Also, it is possible to determine the contribution to computed properties from each of the PCFs. What is lost is some variational freedom in the coefficients when going from an expansion of CSFs to an expansion over reference CSFs and PCFs. Below we describe the biorthogonal transformation at heart of the method.

\section{Biorthogonal transformations}

In the above context, solving the generalized eigenvalue problem (4) of dimension $(g+p)$ in the basis of the $g$ reference CSFs and $p$ PCFs

$$
\left\{\left|\Phi_{1}^{r}\right\rangle,\left|\Phi_{2}^{r}\right\rangle, \ldots\left|\Phi_{g}^{r}\right\rangle,\left|\tilde{\Lambda}_{1}\right\rangle,\left|\tilde{\Lambda}_{2}\right\rangle, \ldots\left|\tilde{\Lambda}_{p}\right\rangle\right\}
$$

requires the calculation of the Hamiltonian and Gram (overlap) matrix elements. In the present approach, freezing the orbitals optimized for the MCHF reference function guarantees the orthononormality of orbitals subsets involved in $\left\langle\Phi_{i}^{r}|H| \tilde{\Lambda}_{j}\right\rangle$ and $\left\langle\Phi_{i}^{r} \mid \tilde{\Lambda}_{j}\right\rangle$ matrix elements, but one-electron nonorthogonalities definitely appear in off-diagonal matrix elements coupling different PCFs, i.e. $\left\langle\tilde{\Lambda}_{i}|H| \tilde{\Lambda}_{j}\right\rangle$. In the most general case invoking other optimization strategies, oneelectron nonorthogonalities will show up in all but the diagonal matrix elements. The biorthonormal approach is then applied for evaluating such matrix elements.

The biorthogonal transformation is explained in details in [26] focusing on the calculation of transition probabilities using nonorthogonal orbitals. This method has shown to be very efficient and useful, producing reliable atomic transition data [41, 42] through the atomic structure package ATSP2K [43]. It has also been implemented in GRASP2K [44] for the calculation of transition amplitudes in the full relativistic context [16]. The idea is simple: two orbital sets that are not orthonormal to each other are first transformed to become biorthonormal. For a coupling matrix element $\left\langle\tilde{\Lambda}_{l}|H| \tilde{\Lambda}_{r}\right\rangle$ built in their own orbital basis $\left\{\phi_{i}^{L}\right\}$ and $\left\{\phi_{i}^{R}\right\}$ that are not orthonormal,

$$
\left\langle\phi_{i}^{L} \mid \phi_{j}^{R}\right\rangle=S_{i j}^{L R},
$$

linear transformations ${ }^{5}$

$$
\phi^{A}=\phi^{L} \mathbf{C}^{L A} ; \quad \phi^{B}=\phi^{R} \mathbf{C}^{R B},
$$

are found to transform the two original orbital sets into two new biorthonormal sets

$$
\left\langle\phi_{i}^{A} \mid \phi_{j}^{B}\right\rangle=\delta_{i j} .
$$

\footnotetext{
5 In our matrix notation, the orbitals $\left\{\phi_{i}\right\}$ are collected in row vectors $\phi$.
}

The advantage of the biorthonormality property (22) of the transformed orbital sets is that the evaluation of any matrix element can proceed as in the orthonormal case, as originally found by Moshinsky and Seligman [45]. There is an infinity of pairs of transformation matrices $\left(\mathbf{C}^{L A}, \mathbf{C}^{R B}\right)$ that produce biorthonormal basis sets. In our approach [26], the choice adopted is predicted by the restrictions on the configuration state function spaces used for $\tilde{\Lambda}_{l}$ and $\tilde{\Lambda}_{r}$. We require the transformation matrices to be upper triangular, a suitable choice for estimating the effect of the orbital transformation on the mixing coefficients $\left\{\alpha_{i}^{L}\right\} \rightarrow\left\{\alpha_{i}^{A}\right\} /\left\{\alpha_{i}^{R}\right\} \rightarrow\left\{\alpha_{i}^{B}\right\}$ giving the two representations of the $\left|\tilde{\Lambda}_{l}\right\rangle$ and $\left|\tilde{\Lambda}_{r}\right\rangle$ functions in both original and transformed (biorthonormal) basis sets

$$
\begin{aligned}
& \left|\tilde{\Lambda}_{l}\right\rangle=\sum_{i} \alpha_{i}^{L}\left|\Phi_{i}^{L}\right\rangle=\sum_{i} \alpha_{i}^{A}\left|\Phi_{i}^{A}\right\rangle, \\
& \left|\tilde{\Lambda}_{r}\right\rangle=\sum_{i} \alpha_{i}^{R}\left|\Phi_{i}^{R}\right\rangle=\sum_{i} \alpha_{i}^{B}\left|\Phi_{i}^{B}\right\rangle .
\end{aligned}
$$

Malmqvist [25] has indeed shown that an upper-triangular orbital transformation matrix can be expressed as a finite sequence of single-orbital transformations, each expressing the new orbitals as a sum involving no higher numbered orbital. Each such transformation step on the CI-expansion array is the same as the effect of a one-electron operator with deexcitations only. To avoid symmetry-breaking intermediates in the recursive transformation, some atomic symmetry refinement was made in [26] to express the transformation operator acting in the CSF space in the following suitable form

$$
\left(\sum_{N=0}^{2(2 l+1)} \frac{1}{N} \hat{\mathrm{s}}^{N}\right) t_{n n}^{\hat{N}_{n}}
$$

with

$$
\hat{s}=\sum_{n^{\prime} \neq n} \frac{t_{n^{\prime} n}}{t_{n n}}\left(\sum_{m_{l} m_{s}}^{2(2 l+1)} a_{n^{\prime} l m_{l} m_{s}}^{\dagger} \hat{a}_{n l m_{l} m_{s}}\right) .
$$

$\hat{N}_{k}$ is the occupation number of the $(n l)_{k}$-subshell and $\mathbf{t}$ is the matrix defining the single orbital replacements sequence that can be calculated from the UL decomposition of $\left(\mathbf{S}^{L R}\right)^{-1}$. The knowledge of the action of the excitation operators in the CSF spaces for both $\left|\tilde{\Lambda}_{l}\right\rangle$ and $\left|\tilde{\Lambda}_{r}\right\rangle$,

$$
\left(\sum_{m_{l} m_{s}}^{2(2 l+1)} a_{n^{\prime} l m_{l} m_{s}}^{\dagger} \hat{a}_{n l m_{l} m_{s}}\right)\left|\Phi_{i}\right\rangle=\sum_{j} A_{i j}\left|\Phi_{j}\right\rangle,
$$

is then enough to perform the countertransformations of the corresponding CI vectors. Starting from the coupled tensorial second quantized form of the single-particle Hamiltonian operator

$$
H=-\sum_{l} \sqrt{2(2 l+1)} \sum_{n^{\prime}, n}\left(a_{n^{\prime} l}^{\dagger} a_{n l}\right)_{00}^{(00)} I_{n^{\prime} l, n l},
$$

one realizes [26] that $A_{i j}=-\sqrt{2(2 l+1)}$ times the coefficient of the $I_{n^{\prime} l, n l}$ integral found in $H_{i j}$ (see also [46]).

There is an important built-in constraint in the algorithm: the wavefunction expansion spaces for both left and right should be 'closed under de-excitation' to allow this class of 
transformation. Restricted active space (RAS) and complete active space (CAS) expansions [47] satisfy this property, i.e. removing one electron from a subshell $n l$ and placing it in any subshell $n^{\prime} l$ of the same spatial symmetry $\left(n^{\prime}<n\right)$ generates a CSF that appears in the original configuration expansion. Other types of expansion can easily be complemented with CSFs to satisfy the closure condition.

\section{Code implementation}

The nonorthogonal pair-correlation method is implemented in a code module extending the ATSP2K package [43]. The module contains routines for checking closure under deexcitation (lscud), performing biorthogonal transformations (biotrans), evaluating Hamiltonian and overlap matrix elements (biomatrix) and solving the generalized eigenvalue problem [48]. Having determined the reference and the paircorrelation functions, removing the reference CSFs from the latter, the calculation proceeds as follows:

loop over reference and pair-correlation
functions
call lscud
call biotrans
call biomatrix
end loop
call generalized eigenvalue solver

To perform the biorthogonal transformation only one-electron coupling coefficients are needed, and thus this part is computationally fast. The subsequent evaluation of the Hamiltonian and overlap matrix elements utilizes standard Racah algebra techniques in the biorthonormal basis. Since there are two orbital sets being biorthogonal, rather than just one set, normal symmetry relations for radial integrals do not hold and integration routines need to be re-designed. The correctness of the implemented method was checked by comparing Hamiltonian and overlap matrix elements with the corresponding ones calculated using Slater determinant algebra in nonorthogonal bases [32,33]. Due to the complexity of the latter method this could only be done for two-, three- and four-electron systems. The correctness of the codes can also be inferred from calculations using artificially rotated orbital sets, where invariances in the representations are used. Tests for different types of systems showed that the biorthonormal transformation is numerically robust, with negligible loss of accuracy for the matrix elements.

\section{Exploring computational strategies}

The beryllium atom may be thought of as the 'benchmark' atom. It is the first neutral system in which the three correlation effects, i.e. valence, core-valence and core correlation, appear and during the years much work has been devoted to understand and accurately describe these effects. Although the goal of the present work is not to get the lowest absolute total energy of the beryllium ground state, it is appropriate to highlight some important contributions with regard to chronology. Byron and Joachain [49] decoupled the original four-electron problem into a series of helium-like equations describing pair correlation between electrons. A combined configuration-interaction-Hylleraas-type wavefunction study was performed by Sims and Hagstrom [50]. A rather complete correlation study of Be was presented by Froese Fischer and Saxena [23] who introduced the separated-pair MCHF approach (this latter work is undoubtedly the inspiration source of the present approach). Elaborate and highly accurate $\mathrm{Be}$ ground state total energies were predicted by Bunge [51]. An iterative numeric procedure [52] was used to obtain pair functions applied to two-electron systems. Numerical many-body perturbation calculations on Be-like systems were proposed by Salomonson et al [53] adopting a multiconfigurational model space. A unified approach combining the multiconfiguration Hartree-Fock method and many-body perturbation theory was attempted by Morrison $[31,54,55]$. The coupled-cluster single- and double-excitation equations were solved numerically by Salomonson and Öster [56]. After the beryllium atom was 'revisited' by a number of groups [57], the nonrelativistic total energy of the Be ground state was estimated by Lindroth et al [58]. Accurate values for the nonrelativistic total energy of Be ground state have been obtained using the full-core plus correlation method [59]. A new correlation study by Froese Fischer [17] was presented in the MCHF scheme, extending the study of $n$ expansion methods to four-electron systems. Energy levels and oscillator strengths were calculated by Weiss [60] adopting a multireference superposition-of-configurations approach for describing core-correlation effects. Finite-element MCHF and GTO basis set expansions coupled with MRSDCI were used to estimate the beryllium electron affinity by Olsen et al [61]. Although difficulties appear in computing matrix elements in the Hylleraas basis [62, 63], Hylleraas configurationinteraction calculations were performed on the nonrelativistic ground-state energy of the Be atom [64]. The most accurate energies for the ground state of the beryllium-like atoms have been obtained using the exponentially correlated Gaussian basis sets $[65,66]$. The most recent work [66] reports the up-to-date lowest infinite mass nonrelativistic total energy of $-14.667356486 E_{\mathrm{h}}$.

Below we explore different computational strategies for $1 s^{2} 2 s^{2}{ }^{1} S$ in beryllium based on separately optimized pair-correlation functions. The results are compared with calculations utilizing a single orthonormal orbital set. Whereas many accurate computational schemes can only be applied to small systems, the current method is directly generalizable to more complex systems and cases for which it is currently not possible to saturate the orbital basis (see section 9).

Monoreference $1 \mathrm{~s}^{2} 2 \mathrm{~s}^{2}{ }^{1} \mathrm{~S}-(4 \times 4)$ approach. We start by generating the three separate PCFs: the valence, the corevalence, and the core-core:

$$
\begin{aligned}
& \left|\Lambda_{\mathrm{VV}}\right\rangle=\alpha_{1}\left|1 \mathrm{~s}^{2} 2 \mathrm{~s}^{2}{ }^{1} \mathrm{~S}\right\rangle \\
& +\sum_{n} \alpha_{n}\left|1 \mathrm{~s}^{2} 2 \mathrm{~s} n \mathrm{~s}{ }^{1} \mathrm{~S}\right\rangle+\sum_{n l, n^{\prime} l^{\prime}} \alpha_{n l, n^{\prime} l^{\prime}}\left|1 \mathrm{~s}^{2} n l n^{\prime} l^{\prime}{ }^{1} \mathrm{~S}\right\rangle
\end{aligned}
$$

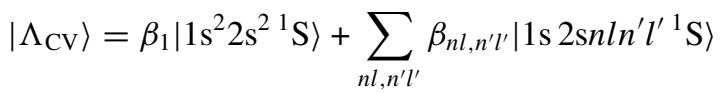


Table 1. Energies together with the number of CSFs for the valence (VV), core-valence (CV) and core-core (CC) PCFs. The (1s, $\underline{2 \mathrm{~s}})$ orbitals are from HF. Correlation orbitals for each of the PCFs are determined in separate MCHF calculations.

\begin{tabular}{crrlrlr}
\hline$n \leqslant$ & \multicolumn{1}{l}{$\mathrm{E}_{\mathrm{VV}}$} & $N_{\mathrm{CSF}}$ & \multicolumn{1}{l}{$\mathrm{E}_{\mathrm{CV}}$} & $N_{\mathrm{CSF}}$ & $\mathrm{E}_{\mathrm{CC}}$ & $N_{\mathrm{CSF}}$ \\
\hline $\mathrm{HF}$ & -14.57302317 & 1 & -14.57302317 & 1 & -14.57302317 & 1 \\
2 & -14.61606266 & 2 & -14.57423722 & 2 & -14.59515832 & 2 \\
3 & -14.61861953 & 7 & -14.57806234 & 6 & -14.61226639 & 7 \\
4 & -14.61899008 & 16 & -14.57878157 & 19 & -14.61439807 & 16 \\
5 & -14.61908321 & 30 & -14.57896023 & 40 & -14.61504150 & 30 \\
6 & -14.61912118 & 50 & -14.57902181 & 72 & -14.61531590 & 50 \\
7 & -14.61913874 & 77 & -14.57905002 & 117 & -14.61545515 & 77 \\
8 & -14.61914803 & 112 & -14.57906466 & 177 & -14.61553036 & 112 \\
9 & -14.61915381 & 156 & -14.57907126 & 254 & -14.61558019 & 156 \\
10 & -14.61915724 & 210 & -14.57907687 & 350 & -14.61561188 & 210 \\
\hline
\end{tabular}

Table 2. Solution of the $(4 \times 4)$ generalized eigenvalue problem. The energies are compared with the SD-MCHF results based on a single orthonormal orbital set.

\begin{tabular}{rllllll}
\hline$n \leqslant$ & $c_{1 \mathrm{~s}^{2} 2 \mathrm{~s}^{2}}$ & $c_{\mathrm{VV}}$ & $c_{\mathrm{CV}}$ & $c_{\mathrm{CC}}$ & $E_{4 \times 4}$ & $E_{\mathrm{SD}-\mathrm{MCHF}}$ \\
\hline 2 & 0.955006 & 0.294901 & $1.1855[-2]$ & $2.9271[-2]$ & -14.63685203 & -14.61685226 \\
3 & 0.959405 & 0.278621 & $2.1230[-2]$ & $3.8235[-2]$ & -14.65828146 & -14.65117428 \\
4 & 0.959708 & 0.277380 & $2.2430[-2]$ & $3.8948[-2]$ & -14.66129822 & -14.65784472 \\
5 & 0.959832 & 0.276919 & $2.2651[-2]$ & $3.9046[-2]$ & -14.66217117 & -14.66093003 \\
6 & 0.959920 & 0.276607 & $2.2714[-2]$ & $3.9061[-2]$ & -14.66252785 & -14.66186116 \\
7 & 0.959978 & 0.276406 & $2.2737[-2]$ & $3.9062[-2]$ & -14.66270455 & -14.66241374 \\
8 & 0.960006 & 0.276305 & $2.2746[-2]$ & $3.9059[-2]$ & -14.66279945 & -14.66274211 \\
9 & 0.960024 & 0.276246 & $2.2750[-2]$ & $3.9057[-2]$ & -14.66285890 & -14.66290834 \\
10 & 0.960035 & 0.276206 & $2.2752[-2]$ & $3.9055[-2]$ & -14.66289785 & -14.66301315 \\
\hline
\end{tabular}

$$
\begin{aligned}
\left|\Lambda_{\mathrm{CC}}\right\rangle & =\gamma_{1}\left|1 \mathrm{~s}^{2} 2 \mathrm{~s}^{2}{ }^{1} \mathrm{~S}\right\rangle \\
+ & \sum_{n} \gamma_{n}\left|1 \mathrm{~s} 2 \mathrm{~s}^{2} n \mathrm{~s}{ }^{1} \mathrm{~S}\right\rangle+\sum_{n l, n^{\prime} l^{\prime}} \gamma_{n l, n^{\prime} l^{\prime}}\left|2 \mathrm{~s}^{2} n l n^{\prime} l^{\prime}{ }^{1} \mathrm{~S}\right\rangle
\end{aligned}
$$

The $1 \mathrm{~s}$ and 2 s orbitals are taken from an initial HF calculation and kept frozen. All correlation orbitals are variational resulting in three sets of orbitals. The energies and number of CSFs as a function of the largest principal quantum number are displayed in table 1 for each of the PCFs. The table shows, as expected, larger correlation energies for valence and core excitations, relatively to core-valence.

Denoting the PCFs in which the reference CSF $\left|1 s^{2} 2 s^{2}{ }^{1} S\right\rangle$ has been removed by setting the corresponding expansion coefficient to zero by, respectively, $\left|\tilde{\Lambda}_{\mathrm{VV}}\right\rangle,\left|\tilde{\Lambda}_{\mathrm{CV}}\right\rangle$ and $\left|\tilde{\Lambda}_{\mathrm{CC}}\right\rangle$, we write the wavefunction as

$|\Psi\rangle=c_{1 \mathrm{~s}^{2} 2 \mathrm{~s}^{2}}\left|1 \mathrm{~s}^{2} 2 \mathrm{~s}^{2}{ }^{1} \mathrm{~S}\right\rangle+c_{\mathrm{VV}}\left|\tilde{\Lambda}_{\mathrm{VV}}\right\rangle+c_{\mathrm{CV}}\left|\tilde{\Lambda}_{\mathrm{CV}}\right\rangle+c_{\mathrm{CC}}\left|\tilde{\Lambda}_{\mathrm{CC}}\right\rangle$.

The expansion coefficients and the total energies are obtained by solving the corresponding $4 \times 4$ generalized eigenvalue problem, see table 2 . The total energies are compared with the SD-MCHF results obtained from the 'traditional' approach, i.e. (i) generating the CSF expansion by single- and doubleexcitations from the reference state and (ii) varying a common set of the orbitals representing the three correlation effects. The convergence with respect to the increasing orbital set is initially much faster using the nonorthogonal pair-correlation approach, but as the orbital basis start to saturate the different correlation effects, the traditional method gives a lower total energy. Looking at the results one should bear in mind that for a given active set specified by the largest principal quantum number $n$, the number of variational orbitals is larger by a factor three in the pair-correlation approach, while the size of the PCF expansions is smaller than the traditional SD-MCHF expansion by roughly the same factor.

Multireference $1 s^{2}\{2 s, 2 p\}^{2} \mathrm{~S}-(5 \times 5)$ approach. In this section we investigate a multireference calculation based on the Layzer's complex $1 \mathrm{~s}^{2}\left\{2 \mathrm{~s}^{2}+2 \mathrm{p}^{2}\right\}{ }^{1} \mathrm{~S}$. The CSF list is generated by making simple and double-excitations on this complex to an active set of orbitals. The CSFs are then arranged into the three lists, one for each correlation type:

$$
\begin{aligned}
& \left|\Lambda_{\mathrm{VV}}\right\rangle=\alpha_{1}\left|1 \mathrm{~s}^{2} 2 \mathrm{~s}^{2}{ }^{1} \mathrm{~S}\right\rangle+\alpha_{2}\left|1 \mathrm{~s}^{2} 2 \mathrm{p}^{2}{ }^{1} \mathrm{~S}\right\rangle \\
& \quad+\sum_{n} \alpha_{n}\left|1 \mathrm{~s}^{2} 2 \mathrm{~s} n \mathrm{~s}{ }^{1} \mathrm{~S}\right\rangle+\sum_{m} \alpha_{m}\left|1 \mathrm{~s}^{2} 2 \mathrm{p} m \mathrm{p}^{1} \mathrm{~S}\right\rangle \\
& \quad+\sum_{n l, n^{\prime} l^{\prime}} \alpha_{n l, n^{\prime} l^{\prime}}\left|1 \mathrm{~s}^{2} n l n^{\prime} l^{\prime}{ }^{1} \mathrm{~S}\right\rangle \\
& \quad+\sum_{n l, n^{\prime} l^{\prime}} \beta_{n l, n^{\prime} l^{\prime}}\left|1 \mathrm{~s} 2 \mathrm{~s} n l n^{\prime} l^{\prime}{ }^{1} \mathrm{~S}\right\rangle \\
& \quad+\sum_{n l, n^{\prime} l^{\prime}} \beta_{n l, n^{\prime} l^{\prime}}^{\prime} \mid 1 \mathrm{~s} 2 \mathrm{p} n l n^{\prime} l^{\prime} \mathrm{S}^{1} \mathrm{~S} \\
& \quad \beta_{1}\left|1 \mathrm{~s}^{2} 2 \mathrm{~s}^{2}{ }^{1} \mathrm{~S}\right\rangle+\beta_{2}\left|1 \mathrm{~s}^{2} 2 \mathrm{p}^{2}{ }^{1} \mathrm{~S}\right\rangle \\
& \left.\quad+\Lambda_{\mathrm{CC}}\right\rangle=\gamma_{1}\left|1 \mathrm{~s}^{2} 2 \mathrm{~s}^{2}{ }^{1} \mathrm{~S}\right\rangle+\gamma_{2}\left|1 \mathrm{~s}^{2} 2 \mathrm{p}^{2}{ }^{1} \mathrm{~S}\right\rangle+\sum_{n} \gamma_{n}\left|1 \mathrm{~s} 2 \mathrm{~s}^{2} n \mathrm{~s}{ }^{1} \mathrm{~S}\right\rangle \\
& \quad \sum_{m} \gamma_{m}\left|1 \mathrm{~s} 2 \mathrm{p}^{2} m \mathrm{p}^{1} \mathrm{~S}\right\rangle+\sum_{n l, n^{\prime} l^{\prime}} \gamma_{n l, n^{\prime} l^{\prime}}\left|2 \mathrm{~s}^{2} n l n^{\prime} l^{\prime 1} \mathrm{~S}\right\rangle \\
& \quad+\sum_{n l, n^{\prime} l^{\prime}} \gamma_{n l, n^{\prime} l^{\prime}}^{\prime}\left|2 \mathrm{p}^{2} n l n^{\prime} l^{\prime} \mathrm{S}\right\rangle
\end{aligned}
$$


Table 3. Energies together with the number of CSFs for the multireference $1 \mathrm{~s}^{2}\{2 \mathrm{~s}, 2 \mathrm{p}\}^{2}{ }^{1} \mathrm{~S}$ valence (VV), core-valence (CV) and core-core (CC) PCFs. The $1 \mathrm{~s}, \underline{2 \mathrm{~s}}, 2 \mathrm{p}$ orbitals are from the multireference MCHF calculation. Correlation orbitals (except $2 p$ ) for each of the PCFs are determined in separate $\overline{\mathrm{MC}} \mathrm{HF}$ calculations.

\begin{tabular}{clrlrlr}
\hline$n \leqslant$ & \multicolumn{1}{c}{$\mathrm{E}_{\mathrm{VV}}$} & $N_{\mathrm{CSF}}$ & $\mathrm{E}_{\mathrm{CV}}$ & $N_{\mathrm{CSF}}$ & $\mathrm{E}_{\mathrm{CC}}$ & $N_{\mathrm{CSF}}$ \\
\hline $\mathrm{MR}$ & -14.61684532 & 2 & -14.61684532 & 2 & -14.61684532 & 2 \\
3 & -14.61890591 & 7 & -14.61942625 & 12 & -14.65431144 & 22 \\
4 & -14.61912407 & 16 & -14.62126576 & 40 & -14.65814875 & 58 \\
5 & -14.61920057 & 30 & -14.62167631 & 93 & -14.65904475 & 119 \\
6 & -14.61923408 & 50 & -14.62176658 & 177 & -14.65937326 & 211 \\
7 & -14.61925100 & 77 & -14.62180187 & 298 & -14.65951762 & 340 \\
8 & -14.61926003 & 112 & -14.62181973 & 462 & -14.65960971 & 512 \\
9 & -14.61926573 & 156 & -14.62182819 & 675 & -14.65966296 & 733 \\
10 & -14.61926869 & 210 & -14.62183399 & 943 & -14.65969630 & 1009 \\
\hline
\end{tabular}

Table 4. Solution of the $(5 \times 5)$ generalized eigenvalue problem. The energies are compared with SD-MR-MCHF and CAS-MCHF results based on a single orthonormal orbital set.

\begin{tabular}{|c|c|c|c|c|c|}
\hline$n \leqslant$ & $c_{1 \mathrm{~s}^{2} 2 \mathrm{~s}^{2}}$ & $c_{1 \mathrm{~s}^{2} 2 \mathrm{p}^{2}}$ & $c_{\mathrm{VV}}$ & $c_{\mathrm{CV}}$ & $c_{\mathrm{CC}}$ \\
\hline 3 & 0.952146 & 0.299633 & $4.2579[-2]$ & $1.7789[-2]$ & $3.8845[-2]$ \\
\hline 4 & 0.952674 & 0.297365 & $4.3498[-2]$ & $2.1246[-2]$ & $4.0538[-2]$ \\
\hline 5 & 0.952773 & 0.296950 & $4.3639[-2]$ & $2.1823[-2]$ & $4.0768[-2]$ \\
\hline 6 & 0.952832 & 0.296749 & $4.3642[-2]$ & $2.1922[-2]$ & $4.0805[-2]$ \\
\hline 7 & 0.952864 & 0.296644 & $4.3629[-2]$ & $2.1956[-2]$ & $4.0817[-2]$ \\
\hline 8 & 0.952885 & 0.296577 & $4.3615[-2]$ & $2.1968[-2]$ & $4.0816[-2]$ \\
\hline 9 & 0.952897 & 0.296543 & $4.3603[-2]$ & $2.1972[-2]$ & $4.0814[-2]$ \\
\hline 10 & 0.952902 & 0.296527 & $4.3597[-2]$ & $2.1973[-2]$ & $4.0813[-2]$ \\
\hline$n \leqslant$ & $\mathrm{E}_{5 \times 5}$ & $\mathrm{E}_{\mathrm{MR}-\mathrm{SD}-\mathrm{MCHF}}$ & $\mathrm{E}_{\mathrm{CAS}-\mathrm{MCHF}}$ & & \\
\hline 3 & -14.65888701 & -14.65439979 & -14.65441459 & & \\
\hline 4 & -14.66477435 & -14.66186568 & -14.66140317 & & \\
\hline 5 & -14.66617394 & -14.66472118 & -14.66483993 & & \\
\hline 6 & -14.66662818 & -14.66593859 & -14.66606732 & & \\
\hline 7 & -14.66682648 & -14.66640790 & -14.66654114 & & \\
\hline 8 & -14.66694578 & -14.66672213 & -14.66685741 & & \\
\hline 9 & -14.66701354 & -14.66687664 & -14.66701275 & & \\
\hline 10 & -14.66705614 & -14.66697555 & -14.66711452 & & \\
\hline
\end{tabular}

This kind of formal writing shows us that the CSF list corresponding to the valence correlation is rigorously the same as the one in the single reference case. However, the content of the two other lists changes, it now includes simple, double, triple and quadruple excitations in comparison with the fundamental configuration $1 s^{2} 2 s^{2}{ }^{1} \mathrm{~S}$. Table 3 contains the results of the three independent pair-correlation MCHF calculations in which the $1 \mathrm{~s}, 2 \mathrm{~s}, 2 \mathrm{p}$ orbitals are frozen from the calculation of the multireference. The remaining correlation orbitals are completely variational in all the calculations.

Again, using the notation $\left|\tilde{\Lambda}_{\mathrm{VV}}\right\rangle,\left|\tilde{\Lambda}_{\mathrm{CV}}\right\rangle$ and $\left|\tilde{\Lambda}_{\mathrm{CC}}\right\rangle$ for the PCFs where the weights of the reference CSFs have been set to zero, the wavefunction is written as

$$
\begin{aligned}
|\Psi\rangle & =c_{1 \mathrm{~s}^{2} 2 \mathrm{~s}^{2}}\left|1 \mathrm{~s}^{2} 2 \mathrm{~s}^{2}{ }^{1} \mathrm{~S}\right\rangle+c_{1 \mathrm{~s}^{2} 2 \mathrm{p}^{2}}\left|1 \mathrm{~s}^{2} 2 \mathrm{p}^{2}{ }^{1} \mathrm{~S}\right\rangle \\
& +c_{\mathrm{VV}}\left|\tilde{\Lambda}_{\mathrm{VV}}\right\rangle+c_{\mathrm{CV}}\left|\tilde{\Lambda}_{\mathrm{CV}}\right\rangle+c_{\mathrm{CC}}\left|\tilde{\Lambda}_{\mathrm{CC}}\right\rangle
\end{aligned}
$$

The expansion coefficients and the total energies are obtained by solving the $5 \times 5$ generalized eigenvalue problem. The coefficients and the energies are reported in table 4 as functions of the largest principal quantum number in the expansions. The energies are compared with values from traditional SD-MR-MCHF and CAS-MCHF calculations. In the $4 \times 4$ approach $\left|1 \mathrm{~s}^{2} 2 \mathrm{p}^{2}{ }^{1} \mathrm{~S}\right\rangle$ entered the $\left|\tilde{\Lambda}_{\mathrm{VV}}\right\rangle$ valence $\mathrm{PCF}$, which obtained a comparatively large weight. Separating out $\left|1 \mathrm{~s}^{2} 2 \mathrm{p}^{2}{ }^{1} \mathrm{~S}\right\rangle$ increases the variational freedom through the expansion coefficient $c_{1 \mathrm{~s}^{2} 2 \mathrm{p}^{2}}$, and the valence PCF becomes just a small correction. Looking at the energies we see a dramatic improvement. The present $5 \times 5$ energy for $n=10$ is comparable to the $n=10 \mathrm{CAS}-\mathrm{MCHF}$ energy based on an expansion of more than $650000 \mathrm{CSF}$. The effort for the CAS-MCHF calculation is huge, and the case has to run for days on a cluster. In contrast, generating the pair-correlation functions, constructing the Hamiltonian and overlap matrices and solving the $5 \times 5$ generalized eigenvalue problem is very fast and is easily done on a PC.

Multireference $1 \mathrm{~s}^{2}\{2 \mathrm{~s}, 2 \mathrm{p}, 3 \mathrm{~s}, 3 \mathrm{p}, 3 \mathrm{~d}\}^{2}{ }^{1} \mathrm{~S}-(8 \times 8)$ approach. Here we extended the multireference to the $n=3$ complex $1 \mathrm{~s}^{2}\left\{2 \mathrm{~s}^{2}+2 \mathrm{p}^{2}+3 \mathrm{~s}^{2}+3 \mathrm{p}^{2}+3 \mathrm{~d}^{2}\right\}^{1} \mathrm{~S}$. As in the previous section we treat each component of the multireference as a subspace of the interaction matrix, increasing the dimension of the generalized eigenvalue problem to 8 . The results of the separate pair-correlation MCHF calculations are displayed in table 5. Even if the size of the largest expansion has increased, it is still a very small problem. Comparing with table 3 , we see that it is the core-valence and core correlation PCFs that now give lower energies. 
Table 5. Energies together with the number of CSFs for the multireference $1 s^{2}\{2 \mathrm{~s}, 2 \mathrm{p}, 3 \mathrm{~s}, 3 \mathrm{p}, 3 \mathrm{~d}\}^{2}{ }^{2} \mathrm{~S}$ valence $(\mathrm{VV})$, core-valence $(\mathrm{CV})$ and core-core (CC) PCFs. The $\underline{n l}(n \leqslant 3)$ orbitals are from the multireference MCHF calculation. Remaining correlation orbitals for each of the PCFs are determined in separate MCHF calculations.

\begin{tabular}{clrlrlr}
\hline$n \leqslant$ & \multicolumn{1}{c}{$\mathrm{E}_{\mathrm{VV}}$} & $N_{\mathrm{CSF}}$ & $\mathrm{E}_{\mathrm{CV}}$ & $N_{\mathrm{CSF}}$ & $\mathrm{E}_{\mathrm{CC}}$ & $N_{\mathrm{CSF}}$ \\
\hline $\mathrm{MR}$ & -14.61891467 & 5 & -14.61891467 & 5 & -14.61891467 & 5 \\
4 & -14.61912628 & 16 & -14.62247934 & 70 & -14.65695343 & 153 \\
5 & -14.61920147 & 30 & -14.62366744 & 192 & -14.66054482 & 352 \\
6 & -14.61923470 & 50 & -14.62391877 & 403 & -14.66128599 & 667 \\
7 & -14.61925152 & 77 & -14.62397710 & 721 & -14.66153251 & 1122 \\
8 & -14.61926043 & 112 & -14.62399933 & 1164 & -14.66164287 & 1743 \\
9 & -14.61926616 & 156 & -14.62400907 & 1750 & -14.66170200 & 2555 \\
10 & -14.61926959 & 210 & -14.62401540 & 2497 & -14.66173742 & 3583 \\
\hline
\end{tabular}

Table 6. Solution of the $(8 \times 8)$ generalized eigenvalue problem. The energies are compared with CAS-MCHF results based on a single orthonormal orbital set.

\begin{tabular}{clllll}
\hline$n \leqslant$ & $c_{1 \mathrm{~s}^{2} 2 \mathrm{~s}^{2}}$ & $c_{1 \mathrm{~s}^{2} 2 \mathrm{p}^{2}}$ & $c_{1 \mathrm{~s}^{2} 3 \mathrm{~s}^{2}}$ & $c_{1 \mathrm{~s}^{2} 3 \mathrm{p}^{2}}$ & $c_{1 \mathrm{~s}^{2} 3 \mathrm{~d}^{2}}$ \\
\hline 4 & 0.953103 & 0.295966 & $-4.0663[-2]$ & $5.8264[-3]$ & $-1.7149[-2]$ \\
5 & 0.953210 & 0.295275 & $-4.0808[-2]$ & $5.1629[-3]$ & $-1.6996[-2]$ \\
6 & 0.953281 & 0.295006 & $-4.0807[-2]$ & $5.1175[-3]$ & $-1.6869[-2]$ \\
7 & 0.953322 & 0.294869 & $-4.0783[-2]$ & $5.0807[-3]$ & $-1.6819[-2]$ \\
8 & 0.953342 & 0.294804 & $-4.0769[-2]$ & $5.0626[-3]$ & $-1.6789[-2]$ \\
9 & 0.953353 & 0.294771 & $-4.0763[-2]$ & $5.0516[-3]$ & $-1.6772[-2]$ \\
10 & 0.953362 & 0.294743 & $-4.0758[-2]$ & $5.0409[-3]$ & $-1.6759[-2]$ \\
$n \leqslant$ & $c_{\mathrm{VV}}$ & $c_{\mathrm{CV}}$ & $c_{\mathrm{CC}}$ & $E_{8 \times 8}$ & $E_{\mathrm{CAS}-\mathrm{MCHF}}$ \\
4 & $8.5613[-3]$ & $2.0715[-2]$ & $3.8931[-2]$ & -14.66067948 & -14.66140317 \\
5 & $9.6715[-3]$ & $2.1911[-2]$ & $4.0595[-2]$ & -14.66555346 & -14.66483993 \\
6 & $9.8881[-3]$ & $2.2103[-2]$ & $4.0808[-2]$ & -14.66658283 & -14.66606732 \\
7 & $9.9702[-3]$ & $2.2135[-2]$ & $4.0844[-2]$ & -14.66690587 & -14.66654114 \\
8 & $9.9847[-3]$ & $2.2148[-2]$ & $4.0849[-2]$ & -14.66704786 & -14.66685741 \\
9 & $9.9822[-3]$ & $2.2151[-2]$ & $4.0848[-2]$ & -14.66712276 & -14.66701275 \\
10 & $9.9793[-3]$ & $2.2151[-2]$ & $4.0846[-2]$ & -14.66716808 & -14.66711452 \\
\hline
\end{tabular}

In this case the wavefunction has the form

$$
\begin{aligned}
|\Psi\rangle & =\sum_{n l, n \leqslant 3} c_{1 \mathrm{~s}^{2} n l^{2}}\left|1 \mathrm{~s}^{2} n l^{2}{ }^{1} \mathrm{~S}\right\rangle \\
& +c_{\mathrm{VV}}\left|\tilde{\Lambda}_{\mathrm{VV}}\right\rangle+c_{\mathrm{CV}}\left|\tilde{\Lambda}_{\mathrm{CV}}\right\rangle+c_{\mathrm{CC}}\left|\tilde{\Lambda}_{\mathrm{CC}}\right\rangle .
\end{aligned}
$$

The expansion coefficients and the total energies are obtained by solving the $8 \times 8$ generalized eigenvalue problem. The coefficients and the energies are reported in table 6 as functions of the largest principal quantum number $n$ in the expansions. The energies are compared with values from traditional CASMCHF calculations, which give the lowest possible energies that can be obtained from a single orthogonal orbital set. Even for $n=10$, that is a basis consisting of 53 orbitals in the orthogonal case, the pair-correlation approach gives a lower total energy. The convergence with respect to the largest principal quantum number $n$ is graphically displayed in figure 2. Here one clearly sees the slow saturation rate of the energy for the CAS-MCHF calculation. For larger systems with more subshells, the difference in saturation rate between calculations using nonorthogonal PCFs and traditional calculations built on a single orbital set will be much greater. Note that although the PCF interaction total energy is lower than the CAS-MCHF one, it is still $2.10^{-4} E_{\mathrm{h}}$ above the Stanke et al's result [66]. Remembering that both the PCF- and CAS-MCHF expansions are $l$-truncated $(l<10)$, this is expected due to the slow convergence rate $\left(E_{l}-E_{l-1} \simeq \mathcal{O}(l+1 / 2)^{-4}\right)$ with respect to $l[67]$.

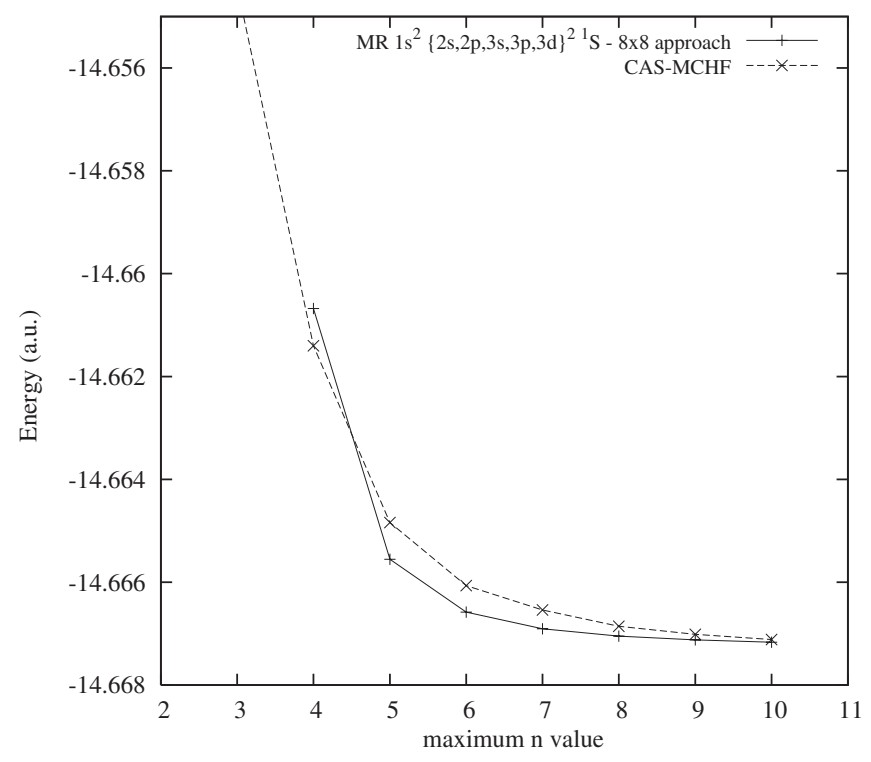

Figure 2. Energies of the $1 s^{2}\{2 s, 2 p, 3 s, 3 p, 3 d\}^{2}{ }^{1} S$ multireference plus VV, CV, CC pair-correlation and the CAS-MCHF expansions as functions of the principal quantum number $n$.

\section{Conclusion and perspectives}

Variational calculations based on 'explicitly correlated Gaussians' are quite impressive but unfortunately scale as $N$ !, 
where $N$ is the number of electrons. As mentioned by Stanke et al [66], calculations with fully correlated basis functions would require huge amounts of computer time for systems with more than six electrons. For the latter, the numerical MCHF method is widely used, generally limited to the optimization of a single orthonormal set of orbitals. However, the relaxation of one-electron orbitals and orthogonality constraints has been shown to be beneficial in many applications $[29,68,69,70]$. The present work demonstrates the effectiveness of relaxing one-electron orthogonality constraints in the MCHF orbital optimization of different PCFs, yielding a new method for treating correlation. Applied to the ground state of beryllium the new method gives total energies that are lower than the ones from traditional CAS-MCHF calculations using large single orbital sets with principal quantum numbers up to 10 . Whereas many accurate computational schemes can only be applied to small systems, the current method is directly generalizable to more complex systems and the cases for which it is currently not possible to saturate a single orbital basis for describing different types of correlation contributing to the total energy, or different type of operators. It is fair to say that we now have the possibility to account for, in a balanced way, correlation deep down in the atomic core in variational calculations.

In this study we have only looked at a few aspects of separately optimized PCFs. Following Froese Fischer and Saxena [23, 24] one could further refine the method and define PCFs for each two-electron coupling. One would then, for example, differentiate between

$$
\begin{aligned}
& \left|\Lambda_{\mathrm{CV}}\right\rangle=\alpha_{1}\left|1 \mathrm{~s}^{2} 2 \mathrm{~s}^{2}{ }^{1} \mathrm{~S}\right\rangle \\
& \quad+\sum_{n l, n^{\prime} l^{\prime}} \alpha_{n l, n^{\prime} l^{\prime}}\left|1 \mathrm{~s} 2 \mathrm{~s}{ }^{1} \mathrm{~S}\left(n l n^{\prime} l^{\prime}{ }^{1} \mathrm{~S}\right){ }^{1} \mathrm{~S}\right\rangle
\end{aligned}
$$

and

$$
\begin{aligned}
& \left|\Lambda_{\mathrm{CV}}\right\rangle=\beta_{1}\left|1 \mathrm{~s}^{2} 2 \mathrm{~s}^{2}{ }^{1} \mathrm{~S}\right\rangle \\
& \quad+\sum_{n l, n^{\prime} l^{\prime}} \beta_{n l, n^{\prime} l^{\prime}}\left|1 \mathrm{~s} 2 \mathrm{~s}^{3} \mathrm{~S}\left(n l n^{\prime} l^{\prime 3} \mathrm{~S}\right){ }^{1} \mathrm{~S}\right\rangle .
\end{aligned}
$$

It would also be possible to introduce some single-electron excitation functions for describing core-opening effects crucial for hyperfine structure and other one-electron operator quantities. The method with separately optimized paircorrelation functions can be extended to a spectrum, describing several states at the same time. The atomic state function is then given by the superposition

$$
|\Psi\rangle=\sum_{r=1}^{M}\left(\sum_{i=1}^{g_{r}} c_{i}^{r}\left|\Phi_{i}^{r}\right\rangle+\sum_{j=1}^{p_{r}} \tilde{c}_{j}^{r}\left|\tilde{\Lambda}_{j}^{r}\right\rangle\right) .
$$

Energies and expansion coefficients of the states are obtained by selected solutions of the eigenvalue problem.

A lot of effort has been put [48] in the general adaptation of ATSP2K [43] for allowing separately optimized PCFs, and it is now possible to compute the expectation of any one- and two-electron operator, including the Breit-Pauli corrections, in the new wavefunction representation. The biorthogonal transformation for handling nonorthogonalities is applicable also in the fully relativistic case. This method with separately optimized PCFs is currently implemented [48] in GRASP2K [44].

\section{Acknowledgments}

SV has a F.R.I.A. fellowship from the F.R.S.-FNRS Fund for Scientific Research. MG thanks the Communauté française of Belgium (Action de Recherche Concertée) and the Belgian National Fund for Scientific Research (FRFC/IISN Convention) for financial support.

\section{References}

[1] Kozlov M G 2004 Int. J. Quantum Chem. 100336

[2] Dzuba V A, Flambaum V V and Kozlov M G 1996 Phys. Rev. A 543948

[3] Dzuba V A and Flambaum V V 2007 Phys. Rev. A 75052504

[4] Safronova M S, Kozlov M G, Johnson W R and Jiang D 2009 Phys. Rev. A 80012516

[5] Lindgren I and Morrison J 1982 Atomic Many-Body Theory (Springer Series on Atoms and Plasmas vol 3) (Berlin: Springer)

[6] Lindgren I and Mukherjee D 1987 Phys. Rep. 293

[7] Li X and Paldus J 2003 J. Chem. Phys. 1195320

[8] Bartlett R J and Musiał M 2007 Rev. Mod. Phys. 79291

[9] Majumder S, Merlitz H, Gopakumar G, Das B P, Mahapatra U S and Mukherjee D 2002 Astrophys. J. 574 513-7

[10] Nataraj H S, Sahoo B K, Chaudhuri R K and Mukherjee D 2007 J. Phys. B: At. Mol. Opt. Phys. 40 3153-62

[11] Sahoo B K and Das B P 2008 Phys. Rev. A 773062516

[12] Roos B O 1987 The complete active space self-consistent field method and its applications in electronic structure calculations Advances in Chemical Physics Ab Initio Methods in Quantum Chemistry II ed K P Lawley (Chichester: Wiley) chapter 69, pp 399-445

[13] Finley J, Malmqvist P-Å, Roos B O and Serrano-Andrés L 1998 Chem. Phys. Lett. 288299

[14] Celani P and Werner H-J 2000 J. Chem. Phys. 1125546

[15] Froese Fischer C, Brage T and Jönsson P 1997 Computational Atomic Structure: An MCHF Approach (Bristol: Institute of Physics Publishing)

[16] Grant I P 2007 Relativistic Quantum Theory of Atoms and Molecules. Theory and Computation (Atomic, Optical and Plasma Physics) (New York: Springer)

[17] Froese Fischer C 1993 J. Phys. B: At. Mol. Opt. Phys. 26 855-62

[18] Godefroid M and Froese Fischer C 1999 J. Phys. B: At. Mol. Opt. Phys. 32 4467-83

[19] Godefroid M and Froese Fischer C 1999 Phys. Rev. A 60 R2637-40

[20] Froese Fischer C 1977 The Hartree-Fock Method for Atoms. A Numerical Approach (New York: Wiley)

[21] Indelicato P 1995 Phys. Rev. A 51 1132-1145

[22] Godefroid M, Jönsson P and Froese Fischer C 1998 Phys. Scr. T 78 33-46

[23] Froese Fischer C and Saxena K M S 1974 Phys. Rev. A 9 1498-1506

[24] Froese Fischer C and Saxena K M S 1975 Phys. Rev. A 12 2281-87

[25] Malmqvist P-Å 1986 Int. J. Quantum Chem. XXX 479-94

[26] Olsen J, Godefroid M, Jönsson P, Malmqvist P- $\AA$ and Froese Fischer C 1995 Phys. Rev. E 52 4499-4508

[27] Hylleraas E A and Undheim B 1930 Z. Phys. 65759

[28] MacDonald J K L 1933 Phys. Rev. 43830

[29] Godefroid M, Van Meulebeke G, Jönsson P and Froese Fischer C 1997 Z. Phys. D 42 193-201

[30] Lindgren I and Salomonson S 1980 Phys. Scr. 21 335-42

[31] Morrison J C and Froese Fischer C 1987 Phys. Rev. A 35 2429-39 
[32] Löwdin P O 1955 Phys. Rev. 97 1474-89

[33] McWeeny R 1992 Methods of Molecular Quantum Mechanics (London: Academic)

[34] Zatsarinny O 1996 Comput. Phys. Commun. 98 235-54

[35] Zatsarinny O and Froese Fischer C 2000 Comput. Phys. Commun. 124 247-89

[36] Fritzsche S, Koike F, Sienkiewicz J E and Vaeck N 1999 Phys. Scr. T 80 479-81

[37] Indelicato P and Desclaux J P 2007 MCDFGME, a multiconfiguration Dirac-Fock and general matrix elements program, release $2007 \mathrm{http}: / /$ dirac.spectro.jussieu.fr/mcdf

[38] Fano U 1965 Phys. Rev. A $14067-75$

[39] Hibbert A, Froese Fischer C and Godefroid M 1988 Comput. Phys. Commun. 51 285-93

[40] Hibbert A and Froese Fischer C 1991 Comput. Phys. Commun. 64 417-30

[41] Froese Fischer C and Tachiev G 2004 At. Data Nucl. Data Tables 87 1-184

[42] Froese Fischer C, Tachiev G and Irimia A 2006 At. Data Nucl. Data Tables 92607

[43] Froese Fischer C, Tachiev G, Gaigalas G and Godefroid M R 2007 Comput. Phys. Commun. 176 559-79

[44] Jönsson P, He X, Froese Fischer C and Grant I P 2007 Comput. Phys. Commun. 177 597-622

[45] Moshinsky M and Seligman T H 1971 Ann. Phys. 66 311-34

[46] Borgoo A, Scharf O, Gaigalas G and Godefroid M 2010 Comput. Phys. Commun. 181 426-39

[47] Olsen J, Roos B O, Jørgensen P and Jensen H J Aa 1988 J. Chem. Phys. 89 2185-92

[48] Jönsson P, Gaigalas G, Godefroid M R and Froese Fischer C 2010 The biopair atomic-structure package for large-scale calculations (in progress)
[49] Byron F W Jr and Joachain C J 1967 Phys. Rev. A 157 162-78

[50] Sims J S and Hagstrom S A 1971 Phys. Rev. A 4908

[51] Bunge C F 1976 Phys. Rev. A 14 1965-78

[52] Mårtensson A-M 1979 J. Phys. B: At. Mol. Phys. 12 3995-4012

[53] Salomonson S, Lindgren I and Mårtensson 1980 Phys. Scr. 21351

[54] Morrison J C 1986 Phys. Scr. 34 423-6

[55] Morrison J C 1988 J. Phys. B: At. Mol. Opt. Phys. 21 2915-21

[56] Salomonson S and Öster P 1990 Phys. Rev. A 414670

[57] Mårtensson A-M, Alexander S A, Adamowicz L, Oliphant N, Olsen J, Öster P, Quiney H M, Salomonson S and Sundholm D 1991 Phys. Rev. A 43 3355-64

[58] Lindroth E, Persson H, Salomonson S and Mårtensson-Pendrill A-M 1992 Phys. Rev. A 45 1493-96

[59] Chung K T, Zhu X-W and Wang Z-W 1993 Phys. Rev. A 471740

[60] Weiss A W 1995 Phys. Rev. A 51 1067-79

[61] Olsen J, Pettersson L G M and Sundholm D 1994 J. Phys. B: At. Mol. Opt. Phys. 27 5575-86

[62] King F W 1993 J. Chem. Phys. 993622

[63] Harris F E, Frolov A M and Smith V H Jr 2004 J. Chem. Phys. 1203040

[64] Büsse G and Kleindienst H 1995 Phys. Rev. A 515019

[65] Pachucki K and Komasa J 2004 Phys. Rev. Lett. 92213001

[66] Stanke M, Komasa J, Bubin S and Adamowicz L 2009 Phys. Rev. A 80022514

[67] Hill R N 1985 J. Chem. Phys. 31173

[68] Froese Fischer C 1973 J. Comput. Phys. 13 502-21

[69] Vaeck N, Godefroid M and Froese Fischer C 1992 Phys. Rev. A 46 3704-16

[70] Zatsarinny O and Froese Fischer C 2000 J. Phys. B: At. Mol. Opt. Phys. 33 313-41 